\title{
Performance hidráulica e perfil de distribuição de água de dois modelos do miniaspersor Supermamkad
}

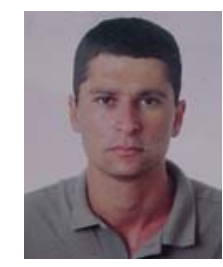

Felizardo A. Rocha1 ${ }^{1}$, Júlio H. F. César ${ }^{1}$, Carlos R. de Mello² \& Geraldo M. Pereira ${ }^{3}$

\author{
1 DEA/UFV. CEP 36570-000, Viçosa, MG. Fone: (31) 3891-8553. E-mail: felizardo.rocha@bol.com.br (Foto) \\ 2 DCS/UFLA. CP 37, CEP 37200-000, Lavras, MG. E-mail: crmello@ufla.br. \\ ${ }^{3}$ Departamento de Engenharia da UFLA. Fone: (35) 3829-1384. E-mail: geraldop@ufla.br
}

Protocolo $068-15 / 5 / 2001$

\begin{abstract}
Resumo: A principal finalidade de ensaios de equipamentos utilizados em sistemas de irrigação por aspersão, consiste na determinação das características hidráulicas e na análise de seu desempenho. O propósito deste trabalho foi avaliar o desempenho de dois modelos do miniaspersor supermamkad, modelo amarelo regulado e não-regulado e violeta não-regulado. Os testes visaram à determinação dos seguintes parâmetros: coeficiente de variação de fabricação (CVF), raio molhado, perfil de distribuição de água e relação vazão versus pressão. Simulou-se também o melhor espaçamento considerando-se as condições utilizadas para hortaliças. As análises mostraram que todos os modelos testados apresentaram o CVF inferior a $5 \%$, o que os classifica como de categoria A. Constatou-se que os emissores podem trabalhar sob pressões de 250, 250 e $300 \mathrm{kPa}$ para os modelos violeta, amarelo regulado e não-regulado, respectivamente, considerando-se espaçamentos típicos de hortículas.
\end{abstract}

Palavras-chave: características hidráulicas, coeficiente de variação de fabricação, uniformidade de distribuição

\section{Hydraulic performance and water distribution profile of two models of the Supermamkad minisprinkler}

\begin{abstract}
The main purpose of testing equipments used in sprinkler irrigation systems is to determine the hydraulic characteristics and to analyze the performance of these equipments. This work aimed at evaluating the performance of two models of the supermamkad minisprinklers, regulated and unregulated yellow models and unregulated violet. The tests sought the determination of the following parameters: water distribution uniformity, manufacturing variation coefficient, mean effective wetted radius and water distribution profile. Also, the best sprinkler spacing for the vegetables was simulated. The analyses have demonstrated that all models tested produced a manufacturing variation coefficient lower than $5 \%$, classifying them in category A. According to simulations, it was possible to conclude that the emitter can work under the pressures of 250, 250 and $300 \mathrm{kPa}$, respectively for the violet and regulated and unregulated yellow models.
\end{abstract}

Key words: hydraulic characteristics, manufacturing variation coefficient, distribution uniformity

\section{INTRODUÇÃO}

A tecnologia de irrigação, desde que adequadamente desenvolvida e/ou adaptada, oferece um instrumento capaz de promover a transformação econômica e social no meio rural, gerando empregos e aumentando a rentabilidade do produtor rural; entretanto, devido às secas cada vez mais freqüentes e duradouras, os irrigantes têm procurado utilizar o recurso água de forma mais eficiente, maximizando a atividade produtiva. $\mathrm{Na}$ irrigação por aspersão, a eficiência da irrigação é um dos principais parâmetros utilizados na sua avaliação, pois expressam a qualidade da irrigação (Rocha et al., 1998).
Mantovani \& Ramos (1994) analisaram a relação entre a lâmina de irrigação e a produtividade para a cultura do milho, e conseguiram, em condições de alta uniformidade $(\mathrm{CUC}=95 \%)$ uma produção de $12.000 \mathrm{~kg} \mathrm{ha}^{-1}$ com uma lâmina de irrigação em torno de $500 \mathrm{~mm}$. Quando a uniformidade foi reduzida para $75 \%$, a lâmina necessária aumentou para valores entre 650 a $700 \mathrm{~mm}$. Em condições de baixa uniformidade $(\mathrm{CUC}=55 \%)$ a lâmina necessária foi superior a $1.100 \mathrm{~mm}$ para alcançar a produção máxima, mostrando que a irrigação pode tornar-se antieconômica.

A importância da uniformidade na agricultura irrigada tem sido documentada por vários pesquisadores. Seginer (1979) e Curtis et al. (1996) demonstraram que um aumento na 
uniformidade de distribuição de água pode aumentar o rendimento das culturas irrigadas e reduzir as perdas por percolação, resultando em benefícios econômicos e ambientais. Assim como a deficiência hídrica pode reduzir o rendimento econômico das culturas, a aplicação excessiva pode resultar em perdas de nutrientes, podendo contaminar os recursos hídricos superficiais e subterrâneos.

Nas últimas décadas, um grande avanço foi observado na irrigação, devido ao surgimento de emissores autocompensantes. Estes emissores possibilitam linhas laterais de grande comprimento com diâmetros reduzidos oferecendo, também, maior uniformidade em áreas extensas e com topografia variável. O mecanismo de autocompensação dos emissores consiste, geralmente, de uma membrana de material flexível inserida em cada emissor, na base de seu bocal; nesta base, existem estrias sobre as quais se assenta a membrana. Em posições da linha lateral em que a pressão da água é elevada, ocorre maior força de assentamento da membrana sobre as estrias, restringindo parcialmente a área de passagem da água para o bocal e a vazão é mantida praticamente constante, devido à redução da área de fluxo. Os valores de vazão são praticamente constantes para uma ampla faixa de pressão (Zanini et al., 1998).

Segundo Solomon (1979) e Vermeiren \& Jobling (1980) o coeficiente de variação de fabricação (CVF) é o parâmetro usado para medir a uniformidade de fabricação dos emissores, expressando bem o projeto do emissor, materiais usados na construção e cuidados aplicados na sua fabricação. Para análise do CVF, a ISO 9260 recomenda uma amostragem aleatória de pelo menos 25 emissores (ISO, 1991).

Villas Boas (1994) comparando o método de amostragem radial com o método de amostragem em malha, verificou que ambos são estatisticamente equivalentes, podendo-se recomendar a utilização do método de amostragem radial para análise da uniformidade de distribuição de água, seja em condições de campo ou na ausência de vento, por ser considerado de instalação fácil e rápida.

A horticultura é a atividade que mais gera e multiplica empregos na agricultura. Cada posto de trabalho na unidade de produção representa cinco outros na cadeia produtiva, de acordo com estatística da Organização das Nações Unidas. Quando se trata de mercado de semipreparados, a ampliação de vagas tem uma progressão geométrica (Tominaga, 2000); entretanto, o consumo per capita de hortaliças no Brasil gira em torno de $45 \mathrm{a} 46 \mathrm{~kg}$ ano ${ }^{-1}$, correspondendo à metade do volume consumido pela Argentina e pouco a mais de um terço do que consomem os EUA e a Europa, porém, o mercado de hortaliças, principalmente as embaladas (fresh cut) é próspero e tende a ocupar fatias cada vez maiores do mercado. Todo este avanço verificado no setor vem acompanhado de técnicas modernas de irrigação.

Dentro do exposto e se considerando a grande aplicação de miniaspersores na irrigação de hortaliças em regiões do tipo "Cinturão Verde" nas proximidades de grandes centros, a finalidade deste trabalho foi avaliar as seguintes características hidráulicas de dois modelos do miniaspersor supermamkad: uniformidade e perfil de distribuição de água, coeficiente de variação de fabricação e raio molhado efetivo, além de simular o melhor espaçamento para uso na horticultura.

\section{MATERIAL E MÉTODOS}

Os ensaios foram realizados no Laboratório de Hidráulica da Universidade Federal de Lavras, em condições de vento nulo, seguindo-se as recomendações das normas ISO 7749/2 (ISO, 1990), ISO 7749/1 (ISO, 1995) e ISO 9260 (ISO, 1991).

Os emissores estudados foram os miniaspersores supermamkad, da empresa Dan Sprinklers, modelos violeta (não-regulado) e amarelo (regulado e não-regulado). Trata-se de um emissor de fabricação israelense, bastante utilizado na irrigação da cultura da banana e em hortaliças; possui estrutura compacta, sendo que o jato de água que entra no emissor aciona uma esfera de aço inoxidável, promovendo seu movimento e, conseqüentemente, o giro do bocal do emissor.

Para a avaliação dos parâmetros mencionados instalou-se uma linha de coletores, espaçados equidistantemente de $1,0 \mathrm{~m}$, a partir do emissor, seguindo-se a metodologia de amostragem radial. Os miniaspersores foram instalados a uma altura de $0,5 \mathrm{~m}$ em relação à seção de captação do coletor.

Para se determinar o coeficiente de uniformidade de Christiansen (CUC), testaram-se três emissores iguais de cada modelo, sendo que os modelos violeta e amarelo não-regulados foram testados nas pressões de 250, 300 e $400 \mathrm{kPa}$ e o modelo amarelo regulado nas pressões de 300, 350, 400, 450 e $500 \mathrm{kPa}$, conforme recomendações do fabricante.

Após uma hora de funcionamento, foram feitas as leituras dos volumes de água contidos em cada coletor, através de uma proveta graduada. No cálculo do CUC, utilizou-se o software Catch-3D, v 4.45, considerando-se a média dos valores de precipitação dos três emissores testados de cada modelo. Esta metodologia foi utilizada para todos os modelos estudados e, a partir da linha resultante, calculou-se a precipitação média para cada pressão de serviço e modelo avaliado.

$\mathrm{O}$ raio efetivo molhado foi considerado como sendo o alcance que proporcionava um volume de pelo menos $1,3 \mathrm{~mL}$ no coletor, o que representa uma intensidade de precipitação de $0,25 \mathrm{~mm} \mathrm{~h}^{-1}$, conforme sugere a norma ISO 7749/1 (ISO, 1995).

Conforme a norma ISO 9260 (ISO, 1991) foram escolhidos, aleatoriamente, 25 emissores de cada modelo, sendo submetidos à pressão de serviço de $300 \mathrm{kPa}$ (pressão recomendada pelo fabricante) para determinação do coeficiente de variação de fabricação, enquanto a vazão do emissor foi determinada pelo método direto, com base na vazão média dos 25 emissores, utilizando-se três repetições por emissor.

Com o emprego do Catch-3D, simulou-se a irrigação da cultura em hortaliças, com o espaçamento entre emissores na linha de 8,10 e $12 \mathrm{~m}$, que corresponde entre 45 e $65 \%$ do diâmetro molhado (Bernardo, 1995) e entre linhas de 10 e $12 \mathrm{~m}$, dentro do intervalo de pressão e espaçamento recomendado pelo fabricante.

\section{RESUTADOS E DISCUSSÃO}

Na Tabela 1 apresentam-se os valores de CUC para o emissor operando isoladamente, com o respectivo raio molhado para cada pressão e modelo do miniaspersor Supermamkad testado.

Observa-se, nesta tabela, que a melhor condição de uniformidade de distribuição de água para os modelos violeta $\mathrm{e}$ amarelo não-regulado, foi alcançada quando esses emissores 
Tabela 1. Valores médios de CUC, raio molhado para cada pressão e modelo do miniaspersor Supermamkad avaliado

\begin{tabular}{|c|c|c|c|c|c|c|}
\hline \multirow{3}{*}{$\begin{array}{c}\text { Pressão } \\
(\mathrm{kPa})\end{array}$} & \multicolumn{3}{|c|}{ CUC Médio - \% } & \multicolumn{3}{|c|}{ Raio Molhado Efetivo - m } \\
\hline & \multicolumn{2}{|c|}{$\mathrm{NRe}$} & \multirow{2}{*}{$\begin{array}{c}\mathrm{Re} \\
\mathrm{Am}\end{array}$} & \multicolumn{2}{|c|}{$\mathrm{NRe}$} & \multirow{2}{*}{$\begin{array}{l}\mathrm{Re} \\
\mathrm{Am}\end{array}$} \\
\hline & $\mathrm{Vi}$ & Am & & $\mathrm{Vi}$ & $\mathrm{Am}$ & \\
\hline 250 & 31,7 & 30,8 & - & 11,0 & 10,0 & - \\
\hline 300 & 30,7 & 24,5 & 40,5 & 11,0 & 10,0 & 9,0 \\
\hline 350 & 21,9 & 12,2 & 42,0 & 11,0 & 10,0 & 9,0 \\
\hline 400 & 20,6 & 20,2 & 42,5 & 11,0 & 10,0 & 9,0 \\
\hline 450 & - & - & 39,3 & - & - & 9,0 \\
\hline 500 & - & - & 40,1 & - & - & 9,0 \\
\hline
\end{tabular}

$\mathrm{NRe}=$ Não-regulado, $\mathrm{Re}=$ Regulado, $\mathrm{Vi}=$ Violeta, $\mathrm{Am}=$ Amarelo

foram submetidos à pressão de $250 \mathrm{kPa}$, enquanto para o modelo amarelo regulado o melhor CUC foi na pressão de $400 \mathrm{kPa}$. Este resultado indica que, para o modelo amarelo, a membrana reguladora de vazão, que é um item opcional, deve ser colocada na base do miniaspersor, pois aumenta expressivamente o coeficiente de uniformidade, elevando-o de 30,8 a 42,5\%. Entretanto, esses dados foram gerados para os emissores operando isoladamente e, em condições de campo, onde o sistema opera com a sobreposição dos jatos de água, o comportamento da uniformidade é alterado, podendo haver elevação da mesma sobre a área irrigada. Portanto, quando os emissores operam em campo com sobreposição dos jatos d'água, os valores de CUC obtidos com o emissor funcionando isoladamente, passam a ter pouca importância, sendo pouco representativos da realidade operacional. De qualquer forma, esta análise individualizada permite uma primeira caracterização do emissor, podendo ser um indicativo do seu melhor desempenho hidráulico.

Ainda pela Tabela 1 nota-se que o raio molhado não foi sensível na faixa de serviço estudada. Os valores deste parâmetro, citados em catálogo para o emissor violeta e amarelo não regulado, são de 9,75 e $9,25 \mathrm{~m}$, à altura de $50 \mathrm{~cm}$, enquanto os valores observados apresentaram raio molhado de 11 e $10 \mathrm{~m}$, respectivamente, sendo ligeiramente superiores.

Na Figura 1 tem-se dados de vazão extraídos de catálogo comparados àqueles observados em laboratório, verificando-se variação máxima de vazão de 2,58, 7,60 e 11,97\%, para os modelos

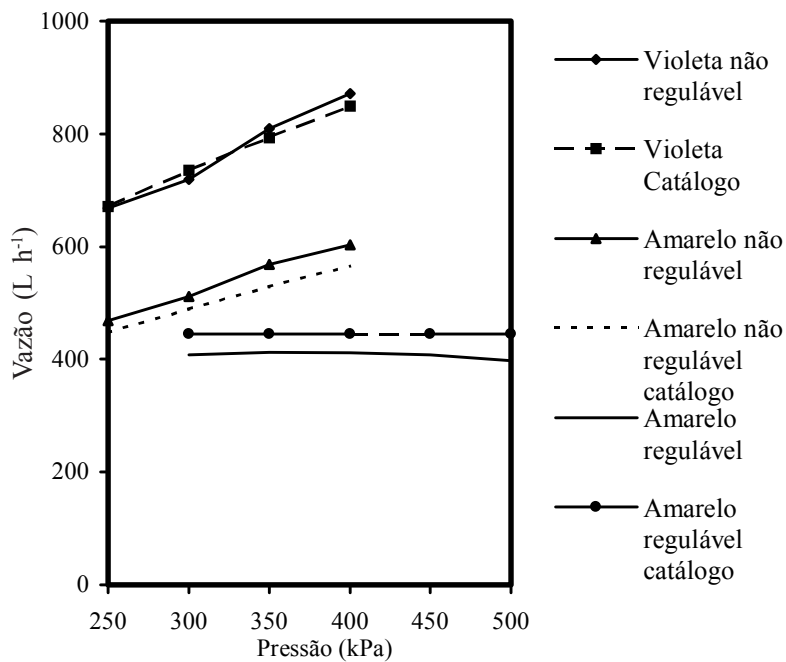

Figura 1. Representação da curva característica de vazão versus pressão para o miniaspersor Supermamkad, obtidas em laboratório e com dados de catálogo violeta, amarelo não-regulado e regulado, respectivamente. Portanto, o emissor violeta apresentou dados coletados mais próximos dos valores de catálogo. Considerando-se apenas os dados observados, a variação máxima de vazão obtida com o aumento da pressão de serviço do emissor foi verificada para o modelo violeta não-regulado (30,32\%); já o emissor amarelo regulado apresentou uma variação máxima de apenas 3,75\% (para menos) com o aumento da pressão, demonstrando que a membrana reguladora de vazão atua restringindo a passagem de água antes de atingir a vazão nominal prevista em catálogo, como pode ser visto na Figura 1.

A ISO 9260/1 (ISO, 1991) considera emissor como de categoria $\mathrm{A}$, aquele que apresenta coeficiente de variação de fabricação (CVF) menor ou igual a 5\%. Sendo assim, pode-se afirmar que os CVF de 2,99, 2,68 e 2,09 encontrados para os modelos violeta, amarelo não-regulado e regulado, respectivamente, permitem classificá-los como de boa qualidade. Uma comprovação disto é demonstrada pelo modelo amarelo regulado, que tem uma precisão quase três vezes maior que a recomendada por esta norma.

Pela Figura 2 vê-se o perfil de distribuição de água apresentado pelos miniaspersores Supermamkad, para as pressões que proporcionaram o melhor CUC, operando isoladamente. Constatou-se que a maior parte do volume de água aplicado pelo modelo violeta não regulado (50,38\%) ocorre nos primeiros $3 \mathrm{~m}$ do seu raio de alcance, contra $41,76 \%$ do modelo amarelo não-regulado e $37,90 \%$ do modelo amarelo regulado. Desta forma, pode-se inferir que a melhor condição de uniformidade de distribuição sobre a área irrigada pode ser encontrada trabalhando-se com o modelo amarelo regulado. Esta falta de uniformidade da irrigação dos emissores não regulados implica em excesso de água em uma parte da área irrigada, podendo acarretar perdas, e sua falta em outra, o que leva à necessidade de aumento da lâmina de irrigação, para se atingir melhores produtividades, conforme Mantovani \& Ramos (1994).

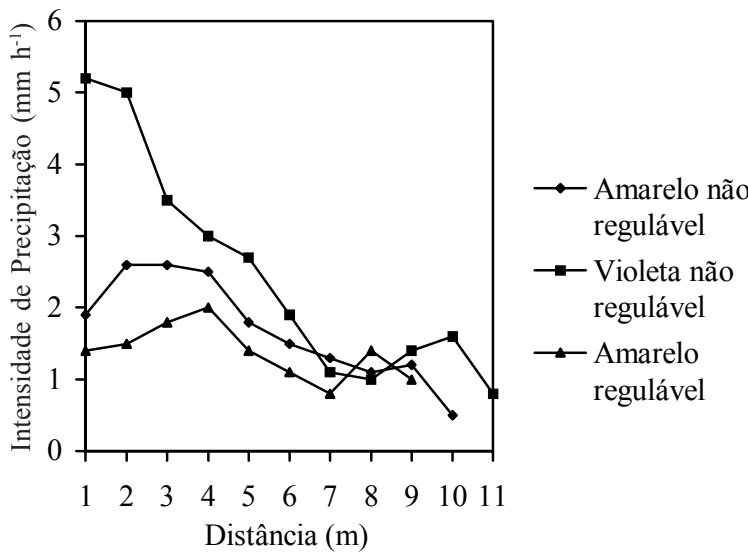

Figura 2. Perfis de distribuição de água do miniaspersor Supermamkad para os modelos violeta, amarelo regulado e não-regulado, operando nas pressões de 250, 400 e $250 \mathrm{kPa}$, respectivamente

Na Tabela 2 têm-se os valores de CUC simulados para a cultura de hortaliças, na qual se verifica que os maiores valores de CUC, após simulação, foram obtidos quando os emissores foram submetidos às pressões de 400, 300 e $300 \mathrm{kPa}$, para os 
Tabela 2. Estimativas de valores de coeficiente da uniformidade do Christiasen (CUC) simulados para a cultura de hortaliças para espaçamentos (E) variados

\begin{tabular}{|c|c|c|c|c|c|c|c|c|}
\hline \multicolumn{3}{|c|}{ Violeta } & \multicolumn{3}{|c|}{ Amarelo não-regulado } & \multicolumn{3}{|c|}{ Amarelo regulado } \\
\hline $\begin{array}{c}\text { Pressão } \\
(\mathrm{kPa})\end{array}$ & $\begin{array}{c}\mathrm{E} \\
(\mathrm{m} \mathrm{x} \mathrm{m})\end{array}$ & $\begin{array}{c}\text { CUC } \\
(\%)\end{array}$ & $\begin{array}{c}\text { Pressão } \\
(\mathrm{kPa})\end{array}$ & $\begin{array}{c}\mathrm{E} \\
(\mathrm{m} \times \mathrm{x})\end{array}$ & $\begin{array}{c}\text { CUC } \\
(\%)\end{array}$ & $\begin{array}{c}\text { Pressão } \\
(\mathrm{kPa})\end{array}$ & $\begin{array}{c}\mathrm{E} \\
(\mathrm{m} \mathrm{x} \mathrm{m})\end{array}$ & $\begin{array}{c}\text { CUC } \\
(\%)\end{array}$ \\
\hline \multirow{5}{*}{250} & $8 \times 10$ & 87,7 & & $8 \times 10$ & 92,8 & \multirow{5}{*}{300} & $8 \times 10$ & 88,6 \\
\hline & $10 \times 10$ & 86,9 & & $10 \times 10$ & 91,9 & & $10 \times 10$ & 88,1 \\
\hline & $10 \times 12$ & 86,8 & 250 & $10 \times 12$ & 89,1 & & $10 \times 12$ & 86,9 \\
\hline & $12 \times 12$ & 84,5 & & $12 \times 12$ & 86,7 & & $12 \times 12$ & 85,6 \\
\hline & $8 \times 10$ & 88,9 & & $8 \times 10$ & 93,9 & & $8 \times 10$ & 87,6 \\
\hline \multirow{4}{*}{300} & $10 \times 10$ & 89,3 & & $10 \times 10$ & 91,9 & \multirow{4}{*}{350} & $10 \times 10$ & 85,7 \\
\hline & $10 \times 12$ & 88,3 & 300 & $10 \times 12$ & 90,9 & & $10 \times 12$ & 83,7 \\
\hline & $12 \times 12$ & 86,0 & & $12 \times 12$ & 89,4 & & $12 \times 12$ & 81,8 \\
\hline & $8 \times 10$ & 92,1 & & $8 \times 10$ & 92,9 & & $8 \times 10$ & 87,8 \\
\hline \multirow{3}{*}{350} & $10 \times 10$ & 91,0 & & $10 \times 10$ & 90,0 & \multirow{3}{*}{400} & $10 \times 10$ & 85,1 \\
\hline & $10 \times 12$ & 91,0 & 350 & $10 \times 12$ & 90,3 & & $10 \times 12$ & 82,5 \\
\hline & $12 \times 12$ & 87,4 & & $12 \times 12$ & 91,1 & & $12 \times 12$ & 81,3 \\
\hline \multirow{4}{*}{400} & $8 \times 10$ & 93,1 & & $8 \times 10$ & 91,4 & \multirow{4}{*}{450} & $8 \times 10$ & 86,8 \\
\hline & $10 \times 10$ & 94,4 & & $10 \times 10$ & 89,8 & & $10 \times 10$ & 85,9 \\
\hline & $10 \times 12$ & 91,9 & 400 & $10 \times 12$ & 88,7 & & $10 \times 12$ & 82,6 \\
\hline & $12 \times 12$ & 88,7 & & $12 \times 12$ & 86,3 & & $12 \times 12$ & 78,0 \\
\hline \multirow{4}{*}{ - } & - & - & & - & - & \multirow{4}{*}{500} & $8 \times 10$ & 87,3 \\
\hline & - & - & & - & - & & $10 \times 10$ & 85,6 \\
\hline & - & - & - & - & - & & $10 \times 12$ & 82,4 \\
\hline & - & - & & - & - & & $12 \times 12$ & 79,4 \\
\hline
\end{tabular}

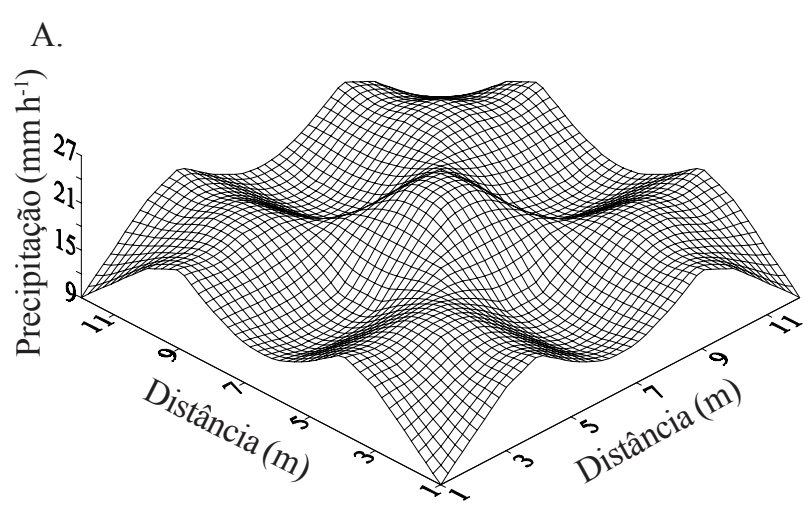

B.

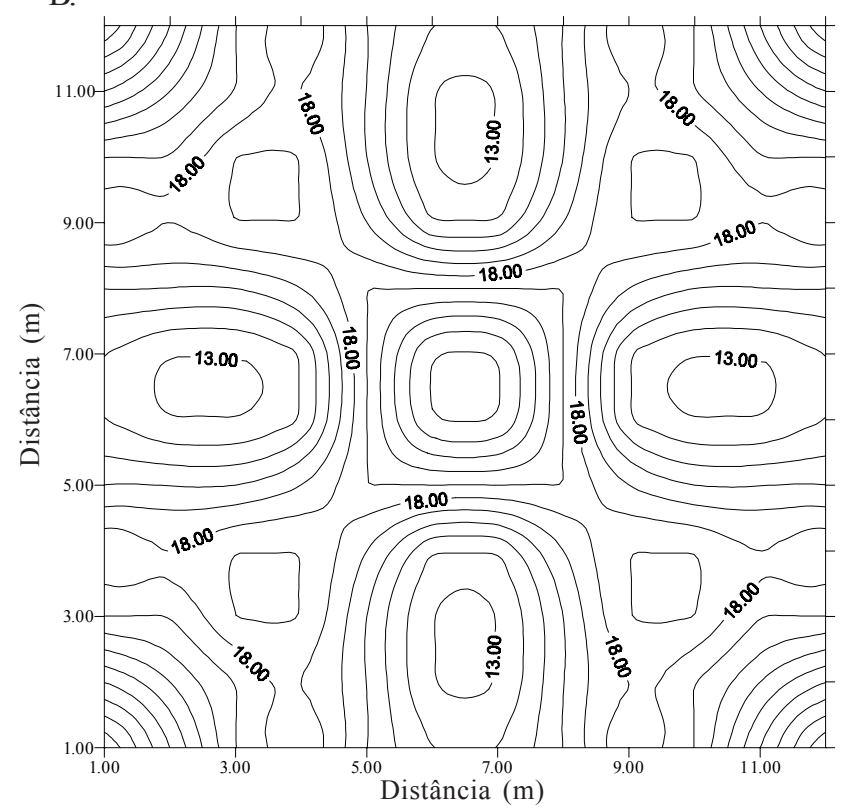

Figura 3. Perfil tridimensional (A) e isoietas (B) do miniaspersor Supermamkad amarelo não-regulado, sob pressão de $250 \mathrm{kPa}$ e espaçamento de $12 \times 12 \mathrm{~m}$ modelos violeta, amarelo regulado e não-regulado, respectivamente.

Como na aspersão valores de CUC entre 80 e $85 \%$ são satisfatórios (Mantovani \& Ramos, 1994) e que para culturas cujo sistema radicular seja pouco profundo, recomenda-se que este coeficiente fique acima de $85 \%$ (Frizzone, 1992) e se pode afirmar que o emissor poderia trabalhar nas pressões de 250 , 250 e $300 \mathrm{kPa}$, para os modelos violeta, amarelo regulado e não-regulado, respectivamente (Tabela 2); isto proporcionaria um custo menor na energia de bombeamento, mantendo boa uniformidade de irrigação; além disso, o espaçamento 12 × 12 m seria o mais recomendado para todos os emissores estudados, permitindo redução no custo por área irrigada, sem prejuízo considerável da uniformidade, conforme valores recomendados acima. Portanto, na elaboração de projetos, aspectos técnicos e econômicos devem ser criteriosamente analisados.

As Figuras 3A e 3B apresentam o perfil de distribuição de água e as isoietas, respectivamente, do miniaspersor Supermamkad amarelo não-regulado operando na pressão de serviço de $250 \mathrm{kPa}$, simulando um espaçamento de 12 x $12 \mathrm{~m}$. Observou-se que nas condições testadas esta foi a melhor performance do emissor, ou seja, maior espaçamento e um CUC satisfatório $(85 \%)$. Estes dados confirmam o bom perfil de distribuição de água apresentado por este emissor quando operando isoladamente. Entretanto, dependendo das condições de vento em campo é necessário reduzir o espaçamento visando manter uma boa uniformidade de distribuição de água.

\section{CONCLUSÕES}

1. Os modelos dos miniaspersores avaliados são de boa qualidade, apresentando CVF inferior a 5\%.

2. As vazões dos miniaspersores não foram condizentes com aquelas apresentadas no catálogo do fabricante, havendo uma variação máxima de $11,97 \%$ para o modelo amarelo regulado. 
3. Pela simulação, constatou-se que os emissores poderiam trabalhar nas pressões de 250,250 e $300 \mathrm{kPa}$, para os modelos violeta, amarelo regulado e não-regulado, respectivamente, uma vez que nestas condições têm-se a melhor performance dos emissores, ou seja, maior espaçamento e um CUC satisfatório $(85 \%)$. Estes dados mostram também que perfis de distribuição de água isolados não são, necessariamente, representativos da realidade de campo.

4. Os valores de raio molhado efetivo observado para os emissores foram ligeiramente superiores aos de catálogo, permitindo maiores espaçamentos.

\section{LITERATURA CITADA}

Bernardo, S. Manual de irrigação. 6. ed. Viçosa: UFV, 1995, $657 p$.

Curtis, L.M.; Powell, A.A.; Tyson, T.W. Microirrigation of peaches in the southeast California. Irrigation Journal, New York, v.46, n.2, p.22-26, 1996.

Frizzone, J.A. Irrigação por aspersão: uniformidade e eficiência. ESALQ/USP. Piracicaba, 1992. 53p. Série Didática No. 003

ISO - International Organization for Standardization. ISO 7719/2. Irrigation equipment - Rotating sprinklers - Part 2: Uniformity of distribution and test methods, Switzerland, 1990.6p.

ISO - International Organization for Standardization. ISO 9260/1. Agricultural irrigation equipment - emitters specification and test methods, Switzerland. 1991.6p.

ISO - International Organization for Standardization. ISO 7749/1. Agricultural irrigation equipment - Rotating sprinklers - Part 1: Design and operational requirements, Switzerland. 1995. 12p.
Mantovani, E.C.; Ramos, M.M. Manejo da irrigação. In Quimigação: aplicação de produtos químicos e biológicos via irrigação. Brasília: EMBRAPA, 1994. cap. 5, p.129-158.

Rocha, E.M. de M.; Mapurunga, S.M. da S; Costa, R.N. T.; Castro, P.T. Uniformidade e eficiência de aplicação de água da irrigação por aspersão convencional na superfície e perfil do solo. In: Congresso Brasileiro de Engenharia Agrícola, 27, 1998, Poços de Caldas. Resumos... Poços de Caldas: Sociedade Brasileira de Engenharia Agrícola, 1998. p.286-288.

Seginer, I. Irrigation uniformity related to horizontal extent of root zone. Irrigation Science, Berlin, v.1, p.89-96, 1979.

Solomon, K.H. Manufacturing variation of trickle emitters. Transactions of the ASAE, St. Joseph, v.22, n.4, p.1034-138. 1979.

Tominaga, M. Hortigranjeiros: o produtor paulista ainda detém a melhor tecnologia. Revista A Granja, Brasília, n.614, p.12-23. 2000.

Vermeiren, I.; Jobling, G.A. Localized irrigation. Rome: FAO, 1980. 203p. Irrigation and Drainage Paper, 36.

Villas Boas, M.A. Análise de métodos de amostragem da distribuição espacial de água de aspersores rotativos. Lavras: ESAL, 1994. 121p. Dissertação de Mestrado

Zanini, J.R; Pavani, L.C.; Tarozzo, M. Avaliação da vazão de um sistema de irrigação por microaspersão em cultura de bananeira. In: Congresso Brasileiro de Engenharia Agrícola, 27, 1998, Poços de Caldas. Resumos... Poços de Caldas: Sociedade Brasileira de Engenharia Agrícola, 1998. p.290-292. 